\title{
Schwächen des Repräsentativmodells: Zur Reichweite direktdemokratischer Verfahren
}

\author{
von Heidrun Abromeit
}

\begin{abstract}
Das neue Phänomen des „Wutbürgers“ steht in engem Zusammenhang mit dem Konzept der „Postdemokratie“. Politische Systeme sind in dem Maße demokratisch, in dem sie dem Volk Möglichkeiten zur effektiven Beteiligung an kollektiven Entscheidungen bieten. In modernen repräsentativen Systemen ist dies nicht wirklich der Fall: Intransparente Verhandlungsdemokratien machen es den Wählern unmöglich, Verantwortlichkeiten zuzuschreiben. Entscheidungsprozesse sind insofern zunehmend „privatisiert“, als ,systemrelevante" Akteure die Richtung vorgeben. Das Elitenkartell verfestigt sich zur Autokratie, der sich der Wähler ohnmächtig gegenübersieht. Abhilfe kann hier nur ein direktdemokratisches Widerspruchsrecht schaffen.
\end{abstract}

The new phenomenon of the "furious citizen" is closely related to the concept of "postdemocracy". Political systems are democratic to the extent that they provide opportunities for the people to participate effectively in the process of collective decision-making. Contemporary representative democracies, however, fall short of allowing their citizens to influence policies by casting their votes. They have, on the contrary, developed into intransparent systems of negotiation that leave voters unable to ascribe political responsibility. These systems are increasingly "privatised" by granting overriding influence to economic actors. They have grown into more or less autocratic elite cartels. In order to "bring the people back in", it is essential to grant them direct-democratic veto powers.

\section{Wutbürger und Postdemokratie}

Die Begriffe „Wutbürger“ und „Postdemokratie“ stehen in der öffentlichen Debatte derzeit an prominenter Stelle - letzteres eher bei wissenschaftlichen Gesellschaftsdeutern, ${ }^{1}$ ersteres eher im Kreis der Schlagzeilen-Fabrikanten - dies jedoch so nachhaltig, dass es zum „Wort des Jahres“ gekürt wurde. Es liegt nahe, beide Begriffe in eine empirische Beziehung zueinander zu setzen: die bürgerliche Wut auf das Misslingen der Demokratie. Diejenigen, die das Wort vom „Wutbürger“ in die Welt gesetzt haben, halten den Zorn der Bürger nicht für

1 Vgl. etwa Mouffe, C.: ,Postdemokratie' und die zunehmende Entpolitisierung, in: Aus Politik und Zeitgeschichte B 1-2 (2011), 3-5; Crouch, C.: Postdemokratie, Bonn, 2009; zuletzt Helms, L.: Ist die Bundesrepublik eine „Post-Demokratie“?, in: ZSE, 8/2 (2010), 202-227. 
legitim, sondern schreiben ihn kleinlich-egoistischer Interessiertheit zu und werten die neue Aufgeregtheit im Volk schlicht als Ungehorsam. Die ironische Pointe dabei: Die Bürger hegen ihrerseits den begründeten Verdacht, dass die Politik sich in ihren Entscheidungen eher an den durchaus egoistischen Interessen von „systemrelevanten“ Großunternehmen, Banken und Lobbygruppen orientiert, als das Allgemeinwohl im Blick zu haben. Und das wiederum entspricht genau dem Bild, das Autoren wie Chantal Mouffe und andere von der Postdemokratie zeichnen: dass nämlich die Umsetzung neoliberaler Politik zu einer „Kolonisierung des Staats durch die Interessen von Unternehmen und Verbänden geführt“ habe, Entscheidungen zunehmend außerhalb der traditionellen demokratischen Kanäle gefällt werden und die formalen demokratischen Prinzipien nur noch als Fassade dienen - ein show act, hinter dem das faktische Geschehen verborgen bleibt. ${ }^{2}$

Die Bürger, die mit großer Mehrheit die Kernenergie ablehnen (und dies seit langem), regen sich zurecht auf, wenn die Regierung in direkter Absprache mit den Energiekonzernen die Verlängerung der Laufzeit der Atomkraftwerke beschließt und diesen Beschluss von den willigen Abgeordneten der Koalitionsfraktionen „demokratisch korrekt“ parlamentarisch absegnen lässt. Folgerichtig intensivierten sich die ohnmächtigen Proteste gegen die Castor-Transporte. Einspruchsrechte seitens der Bevölkerung waren in dieser überlebenswichtigen Frage (schließlich steht die Sicherheit des Zwischenlagers Gorleben in Zweifel) nicht vorgesehen, ja laut Grundgesetz - angeblich - nicht einmal erlaubt. Auch in Stuttgart fühlten sich die Bürger von der Entscheidung der Politik und der Deutschen Bahn für das unterirdische Projekt „Stuttgart 21“ buchstäblich überfahren. Zwar war im Planungsprozess eine gewisse Bürgerbeteiligung vorgesehen, aber offenbar nicht gleichberechtigt und nicht mit voller Information; Argumente von Seiten der Bürger gelten in solchen Zusammenhängen gern als „sachfremd“ und werden von den Experten meist negiert oder umgedeutet. Als das ganze Ausmaß und die Tragweite des Projekts bei Beginn der Abrissarbeiten sichtbar wurden, gingen die braven Stuttgarter Bürger auf die Straße und verstiegen sich sogar zu illegalen Aktionen wie der Schlosspark-Besetzung. Die öffentliche Hand reagierte hart und mit Wasserwerfern. Die Politik kannte keine Milde gegenüber den Störern und teilte ihnen barsch mit, dass sie keinerlei Recht hätten, gegen „demokratisch zustande gekommene Entscheidungen“ aufzubegehren. Das schließlich zugestandene „Schlichtungsverfahren“ unter Leitung von Heiner Geißler schlichtete insofern keinen Konflikt, als den Protestierern unmissver- 
ständlich klar gemacht wurde, dass der Planungs- und Bauprozess längst unumkehrbar sei.

Was an dieser Auseinandersetzung besonders interessant ist, ist das (Totschlags-) Argument der ,demokratisch zustande gekommenen Entscheidung“. Was war daran „demokratisch“ - oder: war das Verfahren doch eher postdemokratisch? Meinte die Regierung nicht vielleicht nur: legal zustande gekommen, also durch parlamentarischen Beschluss abgesegnet?

\section{Was ist eigentlich demokratisch?}

An diesem Punkt sollte man zunächst klären, was ein politisches System zu einem ,demokratischen“ macht. Antworten darauf, was Demokratie sei, füllen seit Jahrhunderten die Bibliotheken; die Meinungen darüber gehen weit auseinander - von der Selbstgesetzgebung des Volkes (Rousseau) bis zur „Herrschaft des Politikers“ (Schumpeter). Hier ist nicht der Ort, all dem nachzugehen. ${ }^{3}$ Konzentrieren wir uns auf das Wesentliche: Dem Begriff des Demokratischen nähert man sich am besten, indem man von konkret institutionalisierten Systemen absieht, die Eigenschaft in den Vordergrund rückt und zugleich nach dem Zweck des Ganzen fragt.

Seit über 150 Jahren orientieren sich Politikwissenschaft und Demokratietheorie an Abraham Lincolns Formulierung, ,government by the people and for the people“, die allerdings, sowohl was das by als auch was das for betrifft, viele unterschiedliche Konkretisierungen zulässt. Immerhin stellt sie klar, dass „das Volk“ der Bezugspunkt zu sein hat. Das Volk besteht aus vielen Einzelnen, denen spätestens seit der Aufklärung das - „unveräußerliche“ - Recht auf Freiheit und Selbstbestimmung zugesprochen wird. Dieses Recht gerät kaum in Gefahr, wenn die Einzelnen so leben, dass sie je für sich selbst entscheiden können. Erst wenn für Gesamtheiten (welcher Art auch immer) zu entscheiden ist, also kollektive Entscheidungen notwendig werden, muss dafür Sorge getragen sein, dass das Selbstbestimmungsrecht nicht unter die Räder gerät. Dann muss der Entscheidungsprozess so organisiert werden, dass er auch wirklich „kollektiv“ ist, also in einer Form, die es ermöglicht, dass alle von der Entscheidung Betroffenen an ihm beteiligt sind, also ein Mitspracherecht haben („Kongruenzprinzip“). Eben dies macht das Demokratische aus: Es ist eine Eigenschaft, die kollektiven Entscheidungssystemen (nicht nur staatlichen) anhaftet (oder eben auch nicht). Und

3 Vgl. hierzu Abromeit, H.: Wozu braucht man Demokratie?, Opladen, 2002. 
da zu den Kernprinzipien der Demokratie auch die Gleichheit zählt, ist ebenso dafür Sorge zu tragen, dass alle Betroffenen in gleicher Weise beteiligt, also gleichberechtigt sind. Der Zweck der Demokratie ist demnach, den ,frei und gleich geborenen“"Individuen ihr Recht auf Selbstbestimmung auch unter den Bedingungen kollektiver Entscheidungen weitestmöglich $\mathrm{zu}$ erhalten. Das ist natürlich nur dann der Fall, wenn die vorhandenen Beteiligungsmöglichkeiten auch in effektiver Weise genutzt werden können.

Gerade das letztere Postulat macht die Umsetzung der theoretisch eigentlich einfachen Prinzipien des Demokratischen in die Praxis schwierig - sie steckt voller Tücken. ${ }^{4}$ Am effektivsten ist natürlich das direkte Mitentscheiden aller, das nur in kleinen Einheiten realisierbar ist (griechische polis, schweizerische Landsgemeinde); in großen Einheiten gibt es keine Agora, die hinreichen würde, den Entscheidungsberechtigten und -willigen auch nur annähernd Platz zu bieten. Man sollte sich aber klarmachen, dass es eine Negativversion der gemeinschaftlichen Entscheidung gibt, nämlich ein Einspruchs- bzw. Widerspruchsrecht gegen getroffene Entscheidungen, das diese blockiert und im Idealfall ein Referendum nach sich zieht. Das Modell der repräsentativen Demokratie sieht das im Allgemeinen nicht vor: Hier wird vikarisch (,für das Volk“) entschieden und ist das individuelle Beteiligungsrecht auf die Wahl von Volksvertretern reduziert. Diese - schwache - Beteiligungsvariante verspricht nur unter einer Reihe von restriktiven Bedingungen Effektivität. Das fängt beim gesellschaftlichen $\mathrm{Zu}$ sammenhalt, der kollektiven Identität und der Solidarität in der Gesamtbevölkerung, an; gemeinsam ermöglichen sie die Herausbildung des Vertrauens der Repräsentierten in ihre Repräsentanten, das seinerseits das Lebenselixier des Repräsentativ-Modells ist. Es wird gefestigt durch die Existenz und Aufrechterhaltung stabiler Verbindungen zwischen Wählern und Gewählten. Lösen sie sich auf, ist damit zu rechnen, dass die Repräsentanten sich von den Repräsentierten emanzipieren (principal-agent problem) - eine Gefahr, die sich durch ein hohes Maß an Transparenz im Rahmen der politischen Vorgänge mindern lässt: hoch genug, um nachträgliche Kontrolle zu ermöglichen.

Mindestens so entscheidend sind die Voraussetzungen, die der Parteienwettbewerb erfüllen muss. Ohne Parteien funktioniert das Repräsentationsmodell nicht: Sie sind die Akteure, die die Wahlen organisieren und dem Bürger die Alternativen vorlegen, zwischen denen er wählen kann. Erst solche Alternativen geben

4 Zum Folgenden vgl. Abromeit,H./Stoiber, M.: Criteria of democratic legitimacy, in: Hurrelmann, A./Schneider, S./Steffek, J. (Hg.): Legitimacy in an age of global politics, Basingstoke, 2007, 35-56. 
der Wahl Sinn und Bedeutung (meaningful elections), und es müssen die ,richtigen“ Alternativen sein, indem sie einen Bezug zu den Präferenzen der Bürger haben und die relevanten Fragen politisieren. Wenn etwa die Parteien vor der Wahl zum Europäischen Parlament hauptsächlich Themen politisieren, über die in Berlin und nicht in Brüssel entschieden wird (was bisher gängigerweise der Fall war), dann erscheint eine Beteiligung an der Wahl politisch bedeutungslos und ist folglich überflüssig. ${ }^{5}$

Schließlich führt die Wahl- und Mehrheitsdemokratie nicht in jedem gesellschaftlichen Kontext zum gewünschten (demokratischen) Ergebnis. Ist die Gesellschaft einigermaßen homogen, das politische System einfach strukturiert, die Zahl und Art der in ihm agierenden Akteure überschaubar und begünstigt die Art des Parteienwettbewerbs die aus Großbritannien bekannten manifesto politics, besteht die Chance, dass auch die Beteiligung allein per Wahl effektiv ist. Sieht der Kontext dagegen anders aus, reicht das Medium Wahlen meist nicht aus und werden Korrektive bzw. ergänzende Beteiligungsformen nötig, um eine effektive Beteiligung zu gewährleisten. Ist die Gesellschaft heterogen, verzeichnet sie etwa Minderheiten, die allein nie die Chance haben, zur Mehrheit zu werden, lässt sich der Anspruch auf Selbstbestimmung durch Wahlen allein nicht realisieren, würden die parlamentarischen Vertreter der Minorität doch immer überstimmt. Dem Übel könnte zwar durch Super-Majoritäten abgeholfen werden. Das hätte aber zur Folge, dass die Minderheits-Repräsentanten in umfängliche Kompromissfindungsprozesse eingebunden würden, die für die Wähler nicht durchschaubar sind und deren Resultate mit den ursprünglichen Programmen wenig gemein haben. In ein Elitenkartell integriert, würden sich ihre Repräsentanten zudem über kurz oder lang ihrer Basis entfremden, und die Basis würde das Vertrauen zu ihnen verlieren.

Mit Rücksicht auf das Problem der strukturellen Minderheit(en) sind die politischen Systeme heterogener Gesellschaften gern hochkomplex; die Komplexität der Mehr-Ebenen-Systeme in Bundesstaaten ist nur eine Spielart davon. Sie alle haben den gleichen Nachteil für die Wähler: Ihr Haupt-Charakteristikum sind komplizierte Verhandlungs- und Vermittlungs-Prozesse, die der Bürger kaum oder gar nicht nachvollziehen kann und die ihn rätseln lassen, wer - welche Partei, welcher Politiker - für welche Politik-Ergebnisse verantwortlich ist und wen er durch Nicht-Wiederwahl abstrafen soll. Kurz: Wahlen garantieren hier

5 Vgl. Mair, P.: ,Popular democracy' and the European Union polity, European Governance Papers, C05-03. 
keine effektive Beteiligung. Dasselbe gilt für korporatistische und vergleichbare Systeme, die gesellschaftliche Akteure in die staatliche Entscheidungsfindung einbinden - umso mehr, als es schon gar keine Möglichkeit gibt, per Wahl etwa einen Wirtschaftsführer zur Verantwortung zu ziehen. Generell lässt sich schlussfolgern, dass Wahlen als Beteiligungsform umso ineffektiver werden, je größer die Zahl der an den kollektiven Entscheidungen maßgeblich mitwirkenden Akteure ist. Und genauso generell kann man sagen, dass das Regieren in und mit Elitenkartellen nichts anderes als eine spezielle Form der Autokratie darstellt. Auch Parteiführungen können natürlich ein solches Elitenkartell bilden, ihrer viel beschworenen Konkurrenz zum Trotz, die häufig nur als SchaufensterDekoration dient. Oder sie verselbständigt sich gar zu einer Art Sport, bei dem es (etwa in talkshows) buchstäblich darauf ankommt, besser auszusehen als der Gegner.

Fast all diese Kontextfaktoren, ebenso wie nahezu alle Elitenkartelle, begünstigen die Auswanderung der relevanten politischen Entscheidungen aus den formell zuständigen - ja sogar vorgeschriebenen - Institutionen und damit die Informalisierung der Politik, die nunmehr hinter verschlossenen Türen stattfindet. Das Volk ist aufs staunende Publikum reduziert, das vor der Tür nur das sieht, was es sehen soll. Böses Erwachen ist garantiert - aber niemand ist „verantwortlich“. Gegen solche Entwicklungen gibt es eigentlich nur ein Heilmittel: direktdemokratische Instrumente. Sie - und insbesondere das Referendum als institutionalisiertes Widerspruchsrecht - sind das beste Korrektiv gegenüber der Arroganz von Elitenkartellen und der Selbstvergessenheit komplexer, informeller, undurchsichtiger Verhandlungssysteme. Sie sind geeignet, die zur Erstarrung in Selbstbezogenheit neigenden repräsentativen Systeme zu demokratisieren.

\section{Defizite moderner repräsentativer Demokratien}

1.

Sieht man sich um, sind die repräsentativen Systeme derzeit nicht selten als demokratisch defizitär anzusehen, der Modebegriff der Postdemokratie scheint darum nicht zu weit her geholt. Dass ihre Defizite zum Teil in ihrer eigenen Funktionslogik begründet sind, hat schon Anthony Downs in seiner „ökonomischen Theorie der Demokratie“ (und in Anlehnung an Schumpeter) aufgewiesen. ${ }^{6}$ Dass das repräsentative Modell nicht ohne Parteien funktioniert, 
wurde schon oben angemerkt. Die Parteien konkurrieren miteinander um Wählerstimmen (und das sollen sie ja auch, es ist sozusagen ihre vornehmste Aufgabe) und sind damit in hohem Maß aufeinander bezogen. Unterstellt man - mit Downs - das Axiom des Eigeninteresses, demzufolge die Konkurrenten nicht durch das Ziel motiviert sind, eine bestimmte Politik umzusetzen oder gar Weltverbesserung zu erstreben, sondern durch die Vorteile von Regierungsamt und Machtbesitz (was, wie man allenthalben sieht, eine realistische Prämisse ist), fragt man sich, wie daraus eine befriedigende Beziehung zwischen Wählern und Parteien resultieren kann. Die Logik erweist sich jedoch - im Modell! - als tragfähig, wenn man eine vergleichbar nutzenorientierte Rationalität beim Wähler und vor allem auf beiden Seiten volle Information (der Wähler über die Vorhaben der Parteien, der Parteien über die Nutzenfunktionen der Wähler) unterstellt. Unter solchen idealen Bedingungen wären Regierende und Parteien unter Androhung des Untergangs bzw. der Wahlniederlage gezwungen, politische Programme anzubieten, die den Präferenzen der Wählermehrheit entsprechen. Unglücklicherweise folgt aus der realen Tatsache des informationellen Ungleichgewichts, dass die Politik-Anbieter die Nachfrager (sprich: Wähler) über den Tisch ziehen können. Das gilt umso mehr, als gerade der rationale Wähler den Nutzen aus der für ihn ,richtigen“ Wahlentscheidung mit seinem minimalen Stimmenwert in Beziehung setzt und feststellen muss, dass die Kosten der Sammlung relevanter Informationen den Nutzen weit übersteigen. Als uninformierter Wähler macht er es den Parteien leicht, ihn allein mit Werbung (nach dem Prinzip „Köpfe und Emotionen“) zu berieseln und mit inhaltsleeren Schlagworten zu traktieren.

So liefert Downs den ökonomischen Beweis für die Marginalität des demos in der konkurrenzdemokratischen Politik; sie ist dem System als solchem inhärent. Damit verweist er zugleich das (vor allem im angelsächsischen Bereich verbreitete) Leitbild des responsible party government ins Reich der Chimären. Ebenfalls ökonomisch weist er im Übrigen einen Trend nach, der zumindest bei den Großparteien eine Angleichung ihrer (verbliebenen) Programmatik an die (vermutete) Mehrheitsmeinung der Wahlbürger nahe legt. Letztere sind demnach nicht nur uninformiert, sondern wüssten auch bei höherem Informationsgrad die Parteien kaum zu unterscheiden. Ihre Wahl wird also nicht nur irrational, sondern letztlich überflüssig. Um so selbstbezogener und ungehemmter können sich die Parteien in ihrem von der Wählerschaft weit abgehobenen Raumschiff dem l'art pour l'art ihrer Machtspiele widmen, deren Sinn von außen kaum zu erschließen ist. 


\section{2.}

Kehren wir uns von Downs und der Funktionslogik der Parteiendemokratie ab und wenden wir uns dem Personal zu, das in ihr agiert. Hier haben wir es nämlich seit einigen Jahrzehnten mit einem neuen Akteurs-Typ zu tun. ${ }^{7}$ Die Entwicklung verlief in der Politik in etwa parallel zur Wirtschaft, wo der EigentümerUnternehmer zunächst von der (Schumpeterschen) Führerfigur des dynamischen Entrepreneurs abgelöst wurde und dieser wiederum vom Finanzjongleur, dem Spieler. ${ }^{8}$ In den Parteien dominierten zunächst die Programmatiker (andere würden sagen: die Ideologen) und Reformer. Da laut Schumpeter für die Politiker politische Ziele und Reformprogramme nur „das Material“ ihrer Tätigkeit sind, ${ }^{9}$ reduzierte sich deren Rolle für ihn auf die Führung: Sie sind die „Feldherren“, die ihre Truppen in den Wahl- und parlamentarischen Kampf führen. Im Zeichen zunehmender Professionalisierung der Politik und Arbeitsteilung kann aber auch davon nur noch begrenzt die Rede sein. Statt seine Truppen in den Kampf zu führen, wird der Politiker selbst von „spin-Doktoren“ und Werbemanagern geführt - als ausgestellte Marke, die im Marxschen Sinne zur Charaktermaske gerinnt: Personalisierung der Politik bei gleichzeitiger Entpersonalisierung der Person. Man könnte vom Verschwinden des Politikers sprechen, denn alle wichtigen, ihm zugeschriebenen Funktionen unterliegen einem Prozess des outsourcing. Er muss nur noch den Kopf hinhalten - in Wahlkämpfen, die andere konzipieren, - und die Stimme heben - in Reden, die andere schreiben. Er mag die Macht zu entscheiden genießen, aber er genießt sie besonders, wenn er nicht entscheiden muss, und wenn es doch unumgänglich ist, entscheidet er so, wie andere es ihm sagen.

Der neue Akteur auf dem politischen Markt ist im Grunde der Nicht-Politiker: Verkäufer, nicht Entscheider, Spieler - aber nur im Sinne des Darstellers; er vertritt nicht das Volk, sondern repräsentiert vor ihm - und zwar eine zumeist „exekutivisch und unternehmerisch bereits vorentschiedene Politik“. ${ }^{10}$ Lessenich und Nullmeier bezeichnen übrigens genau dies als Postdemokratie. Der demokratische Wettbewerb wird hier ebenso wie das responsible party government auf den Kopf gestellt: Alternative Angebote kommen kaum noch aufs Tapet (höchs-

7 Vgl. zum Folgenden Abromeit, H.: Gesellschaften ohne Alternativen, in: Brunkhorst, H. (Hg.): Demokratie in der Weltgesellschaft. Soziale Welt Sonderband 18, Baden-Baden, 2009, 35-56, hier 48 ff.

8 Ebd., $45 \mathrm{ff}$.

9 Schumpeter, J.A.: Kapitalismus, Sozialismus und Demokratie, Bern, 1950, 443.

10 Lessenich, S./Nullmeier, F.: Einleitung: Deutschland zwischen Einheit und Spaltung, in: dies. (Hg.): Deutschland - eine gespaltene Gesellschaft, Frankfurt/New York, 2006, 7-27, hier 24. 
tens durch frustrierte Kleinparteien), Streit und Polarisierung toben sich in Nichtigkeiten aus und der ganze „Lärm um Nichts“ wird veranstaltet, weil man vom Wähler nicht zur Verantwortung gezogen werden will. Nicht umsonst klagen Politik und Öffentlichkeit seit längerem über Parteiverdrossenheit; und nicht umsonst regt sich beim Bürger Wut darüber, „da oben“ so wenig ernst genommen zu werden.

\section{3.}

Stimmt die hier vorgetragene Diagnose, sieht sich der moderne Politiker von den meisten ihm zugeschriebenen Rollen „,befreit“. Von inhaltlich-programmatischen Festlegungen befreit ihn sein Wahlkampfteam, sofern es - wie seit längerem gängig - dem Grundsatz no commitments anhängt, der seinerseits auf Downs zurückgeht. Commitments, also konkrete Versprechungen vor der Wahl, sind insofern kontraproduktiv, als sie mindestens so viele Wählergruppen verprellen wie sie überzeugen. Es entsteht also eine mehr oder weniger große Leerstelle, die bloßes Machtstreben und Machtspielchen nur begrenzt zu füllen vermögen, auch wenn die letzteren durchaus viel Zeit und Energie und sogar Geld kosten können. Macht aber will und soll schließlich genutzt werden. Was also füllt das Vakuum?

Das erste Stichwort, das sich hier anbietet, ist das einer Privatisierung der Politik. In das Vakuum drängen die Vertreter von ,systemrelevanten“ (so heißt das in Deutschland neuerdings) Interessenverbänden, Banken und Großunternehmen systemrelevant deshalb, weil man sie, selbst wenn sie auf Grund unverantwortlicher Zockerei selbst Schuld an ihrem Untergang wären, nicht untergehen lassen kann, will man nicht die Wirtschaft als ganze gefährden. Führende Politiker unterhalten natürlich seit langem gute persönliche Beziehungen zu führenden Wirtschaftsvertretern (man denke nur an Adenauer), die ihnen beim Golf, beim gemeinsamen Frühstück oder an der Bar die richtige Richtung suggerieren. Eher neu ist, dass dies heute in aller Offenheit und Öffentlichkeit geschieht und anschließend zügig umgesetzt wird. Relativ neu sind auch offene Erpressungsversuche, so wie im Fall der vier Energiekonzerne, bevor die Bundesregierung endlich die Verlängerung der Laufzeiten der Atomkraftwerke beschloss; sie erfolgte dann ja auch umgehend.

Ein anderes Stichwort ist das der „Berlusconisierung“ der Politik, ${ }^{11}$ das mehr umfasst als Klientelpolitik, nämlich (aber nicht nur) den Triumph der Eigeninte-

11 Den Begriff Berlusconisation prägte der Economist in einem Bericht über Italien, vgl.: The Berlusconisation of Italy, The Economist, 2.5.2009, $27 \mathrm{f}$. 
ressen der Politiker selbst, die sogar in die Form allgemeingültiger Gesetze gegossen werden. Bekanntlich gibt sich Berlusconi alle erdenkliche Mühe, die italienische Justiz zu schwächen (schließlich ist sie - seinem Verständnis nach kommunistisch unterwandert) und immer neue Immunitätsgesetze verabschieden zu lassen, um zu verhindern, dass er selbst wegen zahlreicher Korruptionsfälle und anderer Delikte zur Rechenschaft gezogen wird. Die Abschaffung des Rechtsstaats ist in der Tat ein Projekt, das einem Diktator oder absoluten Monarchen gut anstünde. In Deutschland die Entwicklung immerhin noch nicht so weit gediehen, dass Politiker sich auf ihre Privatinteressen zugeschnittene Gesetze maßschneidern würden. Der Einfluss der Eigeninteressen auf ihre Politikentscheidungen verrät sich bisher eher indirekt - etwa wenn Kanzler Schröder unmittelbar nach seiner Abwahl einen gut dotierten Posten bei der Gazprom einnimmt. Der Fall Roland Koch könnte ähnlich gelagert sein. ${ }^{12}$

Ein weiterer Aspekt der „Berlusconisierung“ ist im übrigen die Abhängigkeit der wichtigsten Medien vom großen Führer. Berlusconi kontrolliert seit geraumer Zeit etwa die Hälfte der italienischen Fernsehprogramme, eine von vier nationalen Zeitungen, eins von zwei Nachrichtenmagazinen und das größte Verlagshaus, ${ }^{13}$ sodass er den Informationsstand und das Denken eines erheblichen Teils der Bevölkerung zu beeinflussen vermag. Kein Wunder, dass viele Italiener die von außen - und von den „Linken“ - kommende Kritik am cavaliere nicht nachvollziehen können und ihn wieder wählen, wenn sie die Gelegenheit dazu haben, sie sind gleichsam durch ihn sozialisiert. Auch eine solche Entwicklung ist in Deutschland bisher zum Glück nicht zu verzeichnen. Die Aufsichtsräte der öffentlichen Fernsehsender sind noch paritätisch besetzt, und die BILD-Zeitung, deren politischer Einfluss nicht zu unterschätzen ist, schießt hin und wieder sogar gegen ihre konservativen Freunde.

\section{4.}

Gleichwohl tendiert die Entwicklung der großen Mehrzahl der repräsentativen Systeme in dieselbe Richtung: Politische Entscheidungen fallen hinter verschlossenen Türen und unter maßgeblichem Einfluss von Privatinteressen - vor allem wirtschaftlicher Eliten -, während der Einfluss der Bürger auf ein Minimum reduziert ist. Sie können lediglich die regierenden Partei(en) abwählen, aber das

12 Der Wechsel zwischen Führungspositionen in Politik und Wirtschaft und damit die enge personelle Verflechtung war in den USA im übrigen von Anbeginn gängig. Vgl. etwa Mills, C.W.: Die amerikanische Elite, Hamburg, 1962.

13 Vgl. The Economist 2.5.2009, 27. 
nützt ihnen wenig, da die Opposition demselben Druck erliegen wird, auch wenn sie vor der Wahl anderes versprochen hat. Zudem gibt es Entscheidungen und Weichenstellungen, die nicht so ohne weiteres revidierbar sind. Übrigens ändert sich an dem Befund kaum etwas, wenn die verschlossenen Türen geöffnet werden, nicht zuletzt deshalb, weil die Kreise, die die Richtung vorgeben, im Allgemeinen nicht zur Rechenschaft gezogen werden können und nicht absetzbar sind.

Kurz: Mit der Demokratiequalität moderner repräsentativer Systeme ist es nicht gut bestellt. Man muss wohl kaum eigens darauf hinweisen, dass die angesprochene Entwicklung im Zeichen der Globalisierung sowie der dogmatischen Herrschaft des Neoliberalismus nahezu unabwendbar war und Spekulationsgeschäfte von „Heuschrecken“ noch heute als Ausweis „effizienter Märkte“ gelten. ${ }^{14}$

\section{Allheilmittel direkte Beteiligung?}

1.

In den oben angeführten Überlegungen zum Demokratiebegriff und den Kontextbedingungen, unter denen demokratische Politik operiert, wurde deutlich, dass repräsentative Systeme nur unter Idealbedingungen dem Volk eine effektive Beteiligung ermöglichen; die meisten - wie jetzt gesehen - tun es nicht. Sie bedürfen korrektiver Mechanismen, um demokratisch zu bleiben oder zu werden. Und die frustrierten „Wutbürger“ rufen immer lauter nach direkter Beteiligung, die in der Tat das einzig brauchbare Korrektiv zu sein scheint. Mit „,deliberativen Gremien“ jedenfalls dürften die Bürger sich gegen die eben geschilderte Übermacht und deren ökonomische Logik der Sachzwänge kaum durchsetzen können. Aber wie effektiv ist direkte Beteiligung wirklich? Vielleicht gibt es unter den erwähnten Kontextfaktoren auch solche, die die Stimme des Volkes von vornherein entwerten.

Kenner der Materie verweisen in diesem Zusammenhang gern auf die Schweiz und auf Kalifornien - derzeit die beiden Gemeinwesen, in denen die meisten Volksabstimmungen stattfinden. Wer sich mehr mit der Schweiz beschäftigt hat, ist geneigt, den Wert direkter Beteiligung hoch einzustufen, während der Kalifornien-Experte eher Schwächen identifizieren wird. Das hat mit der unterschiedlichen Art der Nutzung direktdemokratischer Instrumente zu tun: In der Schweiz werden sie am häufigsten als Widerspruchsrecht, in Kalifornien dagegen als

14 Vgl. dazu Abromeit, H.: Gesellschaften ohne Alternativen, a.a.O., 51 ff. 
Gesetzesinitiative genutzt. Damit einher geht ein unterschiedlicher Grad an Mediatisierung des Volkes: Zwecks Erarbeitung einer Initiative hat sich quasi eine neue Klasse von ,politischen Unternehmern“ herausgebildet, die sich gern von interessierter Seite finanzieren lassen und von der Vorlage bis zur Kampagne alles „managen“. In der Schweiz wird das Referendum zwar oft auch von interessierten Kreisen in Gang gebracht, namentlich von Verbänden und Parteien (hauptsächlich von deren Untereinheiten), doch bilden sich immer wieder ad hoc-Gruppen von Bürgern, die sich selbst organisieren.

\section{2.}

Daraus folgt zum einen, dass die verschiedenen direktdemokratischen Instrumente in mancher Hinsicht unterschiedlich zu bewerten sind. Zum zweiten ergeben sich erhebliche Unterschiede aus der Frage, wer die Sache wie in Gang bringt. Zum dritten spielen Quoren und deren Höhe eine wichtige Rolle. Ganz entscheidend ist - logischerweise -, ob das Ergebnis einer Volksabstimmung die Politik bindet oder nicht. Zu unterscheiden ist zudem nach den Entscheidungsmaterien. Und schließlich kommt es auch hier noch auf den gesellschaftlichen Kontext an.

Die einzelnen Instrumente sind rasch aufgezählt: ${ }^{15}$ (1) das Personalplebiszit, (2) das akklamatorische Sachplebiszit, (3) das konsultative Referendum, (4) das obligatorische Referendum, (5) das fakultative Referendum und (6) die Gesetzesinitiative. Die ersten drei Instrumente sind „von oben“, sprich der politischen Führung initiiert, die Varianten (5) und (6) von den Bürgern. Schon auf den ersten Blick ist zu vermuten, dass der Faktor der auslösenden Instanz höchst relevant ist für die Frage, welche Instrumente den intendierten Zweck erfüllen können, das Volk tatsächlich effektiv zu beteiligen.

Das Personalplebiszit (1) lässt sich in dieser Hinsicht rasch abhaken. Es ist im Hinblick auf die demokratische Zwecksetzung unerheblich und für den Bürger unter ,symbolische Politik“ abzubuchen. Der Nutzen liegt allein auf der Seite des Gewählten, indem es ihn mit einem besonderen Vertrauensvorschuss ausstattet. Das gilt jedenfalls für die Direktwahl des Präsidenten in normalen repräsentativen Systemen.

Beim akklamatorischen, meist aber auch beim konsultativen Referendum (2 und 3) geht es primär um Mobilisierung der Bürger gegen den parteipolitischen Geg-

15 Vgl. zum Folgenden Abromeit, H.: Nutzen und Risiken direktdemokratischer Instrumente, in: Offe, C. (Hg.): Demokratisierung der Demokratie, Frankfurt/NewYork, 2003, 95-110. S. auch Jung, S.: Die Logik der direkten Demokratie, Wiesbaden, 2001. 
ner. Es wird von oben, nämlich von Präsident, Regierung oder Parlamentsmehrheit initiiert; manchmal kann auch eine Parlamentsminderheit, sprich die Opposition darauf dringen. In beiden Fällen wird die öffentliche Debatte über das Thema von Regierung und Parteien strukturiert. Der Kampf der Parteien wird in die jeweiligen Anhängerschaften verlagert: je stärker deren Auftreten und je höher die Parteiloyalität, desto nebensächlicher die eigentliche Streitfrage. Die Abstimmung hat dann eher den Charakter einer Richtungsentscheidung als eines Sachreferendums. Das Volk entscheidet nicht wirklich mit, sondern wird instrumentalisiert, und so nützt dieses Instrument hauptsächlich den Parteien: Im einen Fall sieht sich die Regierungsmehrheit bestätigt, im anderen verbessert die Opposition ihre Chancen bei der nächsten Wahl. Insofern funktioniert dieses Referendum im mehrheitsdemokratischen Sinn.

Ein Mehrheitsinstrument ist auf den ersten Blick auch das obligatorische Referendum (4), das dadurch definiert ist, dass es für bestimmte Entscheidungen zwingend vorgeschrieben ist. Da es quasi eingebaut ist und nicht eigens initiiert werden muss, entfällt die Möglichkeit, es gezielt im Konkurrenzkampf der Parteien einzusetzen - was die Parteien allerdings nicht davon abhalten wird, alle ihnen zur Verfügung stehenden Mittel zu benutzen, um ihre Anhänger zu mobilisieren. Das gilt zumal für die Regierung, die auf die Zustimmung der Bürger in diesem Fall dringend angewiesen ist. Ist das Referendum mit einem Quorum bewehrt, ist die Regierung, die sich ja nicht immer sicher sein kann, dass sich ihre Mehrheit im Volk zuverlässig mobilisieren lässt, gut beraten, auf breite Zustimmung zu zielen. Die entsprechenden Vorlagen werden darum ausgeprägteren Kompromisscharakter tragen als sonst üblich - mit der Zustimmungsbasis verbreitert sich die Inklusivität. Das obligatorische Referendum wirkt darum nicht notwendigerweise mehrheitsdemokratisch, sondern kann einen supermajoritären, konsensdemokratischen Effekt haben.

In diesem Zusammenhang sind einige klärende Worte zur Logik des Abstimmens einzufügen. Sollen Bürger zur Abstimmung schreiten und sich zuvor auch möglichst noch informieren, bedürfen sie entsprechender Anreize. Die Anreize wachsen mit der Relevanz des Stimmrechts (weswegen bei der Abstimmung über Sachfragen der Informationsgrad im Allgemeinen höher ist als beim bloßen Wahlgang $^{16}$ ) und mit der Interessiertheit, also der Intensität der Präferenzen in der jeweiligen Frage. Die Intensität unserer Präferenzen ist am höchsten, wenn es

16 Bohnet, I./Frey, B.: Direct-democratic rules: the role of discussion, in: KYKLOS Vol. 47, 1994, 341353. 
gilt, eine für die eigenen Rechte und Anliegen bedrohliche Entwicklung abzuwehren. Folglich haben die vehementen Gegner einer Vorlage das stärkste Motiv, sich an der Abstimmung zu beteiligen, während die lauen Befürworter dazu tendieren werden, zu Hause zu bleiben. Das erklärt im übrigen die - jedenfalls in der Schweiz - häufig geringen Beteiligungsraten bei Referenden. Das gilt natürlich nur dann, wenn für die Abstimmung keine Quoren vorgesehen sind. In dem Fall haben engagierte Minderheiten die Chance, sich gegen schweigende Mehrheiten durchzusetzen. Gibt es dagegen Quoren, verhalten sich die Gegner genau umgekehrt: Sie bleiben zu Hause, um auf diese Weise die Vorlage zum Scheitern zu bringen. Das liefert zugleich eine Erklärung dafür, dass manche Regierungen bei solchen zwingend vorgeschriebenen Referenden (etwa über EU-Verträge) das Volk mehrfach abstimmen zu lassen - sozusagen so lange, bis es ,richtig“ votiert. Sie müssen ihre Anhängerschaft noch einmal intensivst - und sei es mit Untergangsszenarien - bearbeiten, damit sie willens ist, die Gegner in Schach zu halten.

Anders als das landläufige Vorurteil besagt, sind Referenden (5) also nicht notwendigerweise mehrheitsdemokratische Instrumente - gar solche zur Beförderung einer „Tyrannei der Mehrheit“ -, sondern können genauso gut Instrumente des Minderheitenschutzes sein: Die genaue Klassifikation hängt vom Vorhandensein und der Höhe der Beteiligungsquoren ab. Das fakultative Referendum wäre, mit hohen Quoren bewehrt, genau genommen sinnlos. Als institutionalisiertes Vetorecht, mittels dessen „aus dem Volk heraus“ parlamentarisch beschlossene Gesetze kontestiert werden können, ist es von seiner Logik her ein Minderheitsinstrument. Idealtypischerweise bedarf es zwar eines (niedrigen) Quorums, um es in Gang zu bringen, aber keines Quorums für die Abstimmung - eben damit „interessierte“ Minderheiten die Chance haben, sich gegen die parlamentarische Mehrheit durchzusetzen (die in ihrer Politik natürlich durchaus nicht immer den Präferenzen der Wählermehrheit folgt). ${ }^{17}$ Insofern entfaltet das fakultative Referendum seinen größten Nutzen in segmentierten Gesellschaften, aber auch im (heute verbreiteten) Fall mehrdimensionaler Politik. Es dürfte zudem der einzige gangbare Weg sein, auf der sektoralen Dimension einen Näherungswert an die Kongruenz von Entscheidungsbetroffenheit und - wenigstens negativer - Entscheidungsbeteiligung herzustellen ${ }^{18}$. Schließlich erscheint es heutzutage als probates Mittel, die abgehobene und selbstbezogene Politiker-

17 Der „Idealtyp“ ist hier natürlich die Schweiz.

18 Vgl. dazu Abromeit, H.: Democracy in Europe, Oxford, 1998. 
Kaste auf Bedürfnisse der Bürger aufmerksam zu machen. Die Drohung, dass ein Gesetz revoziert werden kann, mag manchmal Wunder wirken.

Sind die Parteien noch so stark im Volk verankert, dass sie es in ihrem Sinne instrumentalisieren können, verliert das Referendum natürlich seinen Nutzen. In der Schweiz ${ }^{19}$ wird es zwar auch von Bundesparteien eingesetzt, eher aber von Kantonalparteien, die gegen ihre Vertretung in Bern revoltieren. Vielfach genutzt wird es von Interessenverbänden, die sich jedoch, trotz der oben erläuterten Logik des Abstimmens, nicht so ohne weiteres durchsetzen können. Jedem organisierten Interesse steht nämlich eine Art „zweite Marktseite“ gegenüber, deren Motivation gegenläufig ist und sich fallweise aktualisiert; die Mobilisierungskampagne eines Verbandes kann darum völlig kontraproduktiv sein, zumal in jeder Sachfrage meist mehrere Konfliktlinien tangiert werden. Kurz: der Ausgang eines Referendums ist nicht zuverlässig kalkulierbar. Eben diese Unkalkulierbarkeit vermag (wie die Praxis gezeigt hat) den organisatorischen Vorteil ressourcenstarker Gruppen auszugleichen. Wahrscheinlich stellt diese Form des Referendums überhaupt die einzige Chance für die „unorganisierten“ Bürger dar, via ad hoc-Gruppen ihre Stimme im Konzert der organisierten und institutionalisierten Entscheidungsträger zur Geltung zu bringen.

Diese Stimme kann allerdings nur im Negativen sprechen: Sie erreicht lediglich die Entscheidungsblockade. Deshalb schwören viele Befürworter der direkten Demokratie auf die Gesetzesinitiative als Königsweg demokratischer Selbstbestimmung (6). Das Instrument ist jedoch durchaus ambivalent zu bewerten. Ein nicht zu unterschätzender Nutzen für den Bürger liegt darin, dass die Initiative als solche, also die Einforderung eines Gesetzes, geeignet ist, das QuasiMonopol der Politik auf das agenda setting aufzubrechen. Der Nachteil besteht darin, dass der Gesetzgeber der Aufforderung entweder gar nicht nachkommt oder aber in stark veränderter, verwässerter Form, die nicht dem entspricht, was die Initiatoren sich vorgestellt haben. Sie könnten natürlich gleich eine fertige Vorlage liefern, über die das Volk abstimmen soll. Dabei sind zwei gravierende Probleme zu bedenken: Zum einen wird sich eine ad hoc-Gruppe mit dieser Aufgabe häufig überfordert sehen und zum outsourcing an geeignete Fachleute neigen. Das kann, wie oben beim Beispiel Kalifornien erwähnt, einen speziellen Typ von ,politischen Unternehmern“ auf den Plan rufen, die sich auf derlei Aufgaben spezialisieren. Das Resultat ist dann nicht die vorgestellte „Selbstgesetz-

19 Für einen Überblick vgl. Linder, W.: Schweizerische Demokratie, Bern u.a., 1999, Kap. 10. 
gebung" der Bürger; die herkömmliche Art ihrer Mediatisierung wird lediglich durch eine andere Art ersetzt bzw. ergänzt.

Zum anderen stellt sich hier die Mehrheits-Minderheits-Problematik in besonders akzentuierter Weise. Hierzu muss man sich noch einmal die Logik des Abstimmens vor Augen führen, die bewirken kann, dass eine interessierte Minderheit (mit ,intensiven Präferenzen“) der Mehrheit ein Gesetz aufzwingen kann - jedenfalls unter den beiden Voraussetzungen, dass (a) die Abstimmung bindend wirkt und (b) das Quorum relativ niedrig angesetzt wird. Läge es hingegen bei $51 \%$, so wäre das Instrument von vornherein wertlos und den damit verbundenen Aufwand nicht wert. Unterstellt man jetzt auch noch Parteiendominanz und die Opposition hätte ihre Hände im Spiel, dann wäre nicht nur die Funktionslogik der Mehrheitsdemokratie auf den Kopf gestellt, sondern würden die Bürger am Ende doch nur wieder für den Kampf der Parteien instrumentalisiert.

Die Gesetzesinitiative stellt die Anhänger direkter Beteiligung also vor eine schwierige Entscheidung und wirft die Frage der demokratischen Legitimität auf. Es ist nämlich eine Sache, einer Minderheit das Recht zum Widerspruch gegen Entscheidungen einzuräumen, die sie (bzw. ihre Interessen) ganz essentiell tangieren, aber es ist eine ganz andere Sache, einer engagierten Minderheit die Möglichkeit zuzubilligen, an der Mehrheit vorbei für die Gesamtheit gültige Regeln zu beschließen.

Zum Abschluss wäre noch kurz die Frage zu erörtern, in welchen Materien den Bürgern legitimerweise ein Entscheidungsrecht zuzugestehen ist. In Deutschland ist man überzeugt, dass man sie nicht über finanzwirksame Vorlagen entscheiden lassen dürfe, weil sonst die Staatsfinanzen heillos durcheinander gerieten. Das Schweizer Beispiel hat dieses Vorurteil längst widerlegt, ${ }^{20}$ nicht umsonst ist die Schweiz das Land mit der geringsten Staatsquote und niedrigsten Staatsverschuldung. Es ist eher umgekehrt so, dass die Bürger erst lernen, finanzielle Angelegenheiten zu verstehen, wenn sie darüber abstimmen dürfen. Auch das Argument der Komplexität vieler Sachfragen, die die Kompetenz der Bürger übersteige, kann man so nicht stehen lassen, denn ihre Repräsentanten sind häufig ähnlich inkompetent und lassen ihre Position von (meist sehr interessierten) Experten prägen. Im Übrigen haben im 19. Jahrhundert die damals Herrschenden denselben Vorwurf gegen die repräsentative Demokratie erhoben.

20 Vgl. Kirchgässner, G./ Feld, L./Savioz, M.: Die direkte Demokratie, Basel, 1999, Kap. 4. 
In zwei Typen von Materien allerdings ist die direktdemokratische Entscheidung aus demokratietheoretischen Gründen allerdings hochproblematisch: in allen Fragen, die mit gravierenden Wertdifferenzen zwischen Gruppen/Minderheiten zu tun haben (wie etwa in der Kruzifix- oder der Kopftuch-Frage), und in Fragen, die generell die Rechte und Chancen anderer betreffen (etwa: dürfen Ausländer Inländer werden? Frauen das Stimmrecht erhalten?). Der zweite Fall berührt das knifflige Problem, ob der Demos berechtigt ist, darüber zu entscheiden, wer zum Demos gehört und wer nicht. Das ist eine der Aporien der Demokratietheorie, zu der bisher noch niemand eine überzeugende Lösung vorgelegt hat. Auch das schweizerische Bundesgericht hat sich vor einigen Jahren vergebens darum bemüht, aber immerhin geurteilt, dass das Volk eine solche Entscheidung nicht treffen dürfe. ${ }^{21}$ Es fragt sich nur, ob die Entscheidung in demokratietheoretischer Hinsicht legitimer wird, wenn Repräsentanten sie treffen.

\section{3.}

Der Überblick über die Wirkungsweise der verschiedenen direktdemokratischen Instrumente zeigt, dass direkte Beteiligung den repräsentativen Beteiligungsformen nicht generell überlegen und ebenso wenig generell das Allheilmittel gegen die Defizite der Postdemokratie ist. Auf jeden Fall sind die Varianten je nach Kontext, aber auch mit Blick auf ihre Effektivität differenziert zu beurteilen. Von einem wirklichen Beitrag zu bürgerschaftlicher Mitbestimmung kann man nur bei der Gesetzesinitiative und dem fakultativen Referendum sprechen; im ersten Fall stellt sich die Mehrheits-Minderheits-Problematik deutlich schärfer als im zweiten. Hinsichtlich der Abstimmungslogik sind die Instrumente (sofern sie nicht ,von oben“ instrumentalisiert werden können, und natürlich abhängig von den Quoren und deren Höhe) primär Minderheitsrechte, weshalb sie ihre größte Effektivität in der Funktion als Widerspruchsrecht entfalten. Ein solches Recht wird - wie oben argumentiert - zum demokratietheoretisch geradezu unerlässlichen (Ergänzungs-)Verfahren in segmentierten Gesellschaften und hochkomplexen Entscheidungssystemen, aber auch bei (daraus letztlich resultierend) hohem Informalisierungsgrad der Politik - und schließlich beim Abheben des „Raumschiffs Politik“ in der Postdemokratie.

21 Es ging darum, ob in einer Gemeinde im Kanton Fribourg das Volk darüber entscheiden dürfe, welche Ausländer eingebürgert werden können und welche nicht. Das Gericht zog sich auf das Argument zurück, dass dies nicht ad personam geschehen dürfe. 
Es wurde allerdings nun schon mehrfach auf einen weiteren Kontextfaktor hingewiesen, der die Wirksamkeit selbst des Widerspruchsrechts drastisch mindern kann: die Parteiendominanz (incl. der partyness of society nach Katz). Solange die Parteien großen Einfluss auf das Stimmverhalten ihres Fußvolks ausüben, ist das schönste direktdemokratische Instrument im Hinblick auf vermehrte bürgerschaftliche Mit- und Selbstbestimmung wertlos. Dasselbe gälte natürlich auch für die Dominanz anderer gesellschaftlicher Akteure, wie Verbände, Kirchen, Medien etc. In unser „Maß für Demokratie“ ${ }^{62}$ haben wir deshalb „Dominanzstrukturen“ als eigenen Kontextfaktor aufgenommen. Der Fall der Parteiendominanz (die indessen in den meisten politischen Systemen im Rückzug begriffen ist) stellt sich das Problem besonders akzentuiert, nämlich als Paradox: Sie lässt das direktdemokratische Korrektiv besonders notwendig erscheinen - und entwertet es zugleich.

Abschließend sei noch auf zwei Schwachstellen direkter Beteiligung hingewiesen, die man in Rechnung stellen muss: Aus vielen empirischen Studien wissen wir, dass Beteiligungsraten mit dem Bildungsgrad steigen. Das gilt zwar auch für die Wahlbeteiligung, erhöht aber fallweise den Mittelstands-bias der Politik (wie unlängst in Hamburg bei der Abstimmung über die Schulpolitik zu beobachten). Zum anderen prämiert gerade das effektivste Instrument, nämlich das Widerspruchsrecht, tendenziell die „Ewiggestrigen“. Wer den gesellschaftlichen status quo ändern will, sollte also vorsichtig damit umgehen. Dem ist jedoch sogleich hinzuzufügen, dass diejenigen, die seit langem den größten Einfluss auf die Politik ausüben, an Änderungen (sofern es nicht um die „Sozialstaatlichkeit“ geht) nur bedingt interessiert sind.

\section{Fazit}

Als Anfang 2011 das albanische Volk gegen seine Regierung protestierte, erstaunte die ARD-Korrespondentin das Publikum mit der Bemerkung, dass Albanien ein „demokratisches Land“ sei, „keine Diktatur wie Tunesien“ - um im nächsten Satz fortzufahren, das Volk gehe auf die Straße aus Zorn über ,eine politische Klasse, die sich schamlos bereichert“. ${ }^{23}$ Darin verrät sich dieselbe Unsicherheit darüber, was „,demokratisch“ ist, wie in dem Stuttgarter Diktum,

22 Vgl. Abromeit, H.: Die Messbarkeit von Demokratie, in: Politische Vierteljahresschrift 45 (2004), 7393; Abromeit H./Stoiber, M.: Criteria of democratic legitimacy, a.a.O.

23 ARD, Tagesthemen v. 21.1.2011. 
die Entscheidung für „Stuttgart 21“ sei „demokratisch zustande gekommen“ und deshalb durch bürgerschaftlichen Protest nicht revidierbar.

Man darf sich über diese Unsicherheit nicht wundern, sind doch selbst Demokratieforscher von ihr befallen. Seit über 50 Jahren wurden diverse Messinstrumente für Demokratiequalität entwickelt, die nahezu alle unter der gleichen Schwäche leiden: Sie missverstehen Demokratie als ein fixes Ensemble institutioneller Vorkehrungen und orientieren sich überwiegend am angelsächsischen Modell. Da gilt nicht zuletzt das Motto: ein Staat, in dem es kein Misstrauensvotum gibt, ist als tendenziell autokratisch einzustufen. Die Kontextabhängigkeit der Verwirklichungsformen von Demokratie oder auch trade-offs zwischen verschiedenen Beteiligungsarten - indirekt und direkt - werden im allgemeinen nicht berücksichtigt. Das jüngste Beispiel ist das neue „Demokratiebarometer“, das eine Forschergruppe aus Berlin und Zürich entwickelt hat. ${ }^{24}$ Es weist den zusätzlichen Denkfehler auf, die Höhe der Beteiligungsraten als einen wichtigen Indikator für Demokratiequalität zu werten. Deswegen rangiert Belgien auf Rang 3 (von 29) ihrer Skala - dort besteht Wahlpflicht! - und die Schweiz auf Rang 14 - hinter den USA und Deutschland. ${ }^{25}$ Was die Schweiz betrifft, fällt nicht nur die mangelnde Kalkulation der trade-offs zwischen Wahlbeteiligung und der Beteiligung an Abstimmungen auf, sondern auch das fehlende Verständnis für die (oben erläuterte) Logik des Abstimmens. ${ }^{26}$ Das muss insofern verwundern, als auch schweizerische Demokratieforscher beteiligt waren.

$\mathrm{Zu}$ Recht werten die Autoren des Barometers Transparenz und politische Gleichheit als weitere Indikatoren für Demokratiequalität, ebenso wie (traditionellerweise) gegenseitige Kontrollrechte der Verfassungsorgane. Wie oben geschildert, mangelt es daran auf gravierender Weise in allen Postdemokratien. Die Beteiligung des Volkes an der Wahl kann dagegen nirgends etwas ausrichten, es kann das System nicht durchschauen und darf gleichwohl vermuten, dass , die da oben“ im Konzert mit den Mächtigen der Wirtschaft „sich schamlos bereichern“. Die Möglichkeit der Abstrafung von Personen durch Verweigerung der Wiederwahl erweist sich als weitgehend wertlos, wenn die Neu-Gewählten alsbald in demselben Geflecht stecken. Doch es gibt eine Alternative: die Infragestellung von Entscheidungen durch ein Widerspruchsrecht der Bürger. Trotz der oben beschriebenen Schwächen, die direktdemokratische Instrumente unter bestimm-

24 Bühlmann, M./Merkel, W. et al..; vgl. Neue Zürcher Zeitung v. 29.1.2011.

25 Großbritannien kommt übrigens auf Rang 25 ein, knapp vor Polen, Südafrika und Costa Rica.

26 Vgl. Abromeit, H.: Wie demokratisch ist die Schweiz?, in: Neue Zürcher Zeitung v. 10.2.2011 
ten Bedingungen aufweisen, scheint gerade das Widerspruchsrecht des fakultativen Referendums ein probates Mittel zu sein, um die Oligarchie „derer da oben“ fallweise aufzubrechen und auf diesem Weg zu re-demokratisieren. Werden Entscheidungen des öfteren erfolgreich in Zweifel gezogen, könnten die politischen Akteure à la longue sogar bereit sein, aus ihrem Raumschiff auf die Erde zurückzukehren und von vornherein auf die Bedürfnisse im Volk Rücksicht zu nehmen.

Idealisten glaubten eine Weile, dass Konsens- und Mediationsrunden das geeignete Mittel seien, die Postdemokratie etwas demokratischer zu gestalten. Die Erfahrungen damit haben leider gezeigt, dass dies illusionär ist. Die „Wutbürger“ sind wütender als zuvor, wenn sie begreifen, dass sich ,da oben“ niemand an die Ergebnisse der Mediation gebunden fühlt - in der Wirtschaft schon gar nicht, während die Politik beschönigt, beschwichtigt und verschleiert (vgl. etwa das Beispiel der Frankfurter Flughafen-Erweiterung ${ }^{27}$ ). Obendrein machen sie die Erfahrung, dass ihnen ihre Argumente mit dem Sachzwang-Argument (das häufig nicht mehr als ein Rendite-Argument ist) aus der Hand geschlagen werden und ihre Anliegen allenfalls in nachrangigen Detailfragen Berücksichtigung finden (wie am Beispiel Stuttgart zu sehen). Solche Runden fungieren bisher als dürftiges Mäntelchen für von oben durchgesetzte Interessen und sollten eher als symbolische Politik und Beschäftigungstherapie denn als wirkliche Instrumente der Mitgestaltung interpretiert werden. Auf diesem Weg dürften die Defizite der Postdemokratie nicht zu beheben sein - es sei denn, am Ende stünde die Möglichkeit der Drohung seitens der Frustrierten, nun doch ein Referendum in Gang zu setzen.

27 Vgl. etwa Geis, A.: Regieren mit Mediation, Wiesbaden, 2005 